\title{
Combined effect of negative pressure wound therapy and cryo-air therapy on diabetic wound healing
}

\section{Mahmoud Elshazly ${ }^{a}$, Zeinab M. Askary ${ }^{b}$}

${ }^{a}$ Department of Physical therapy for Surgery, Faculty of physical therapy, South Valley University, Qena, Egypt.

${ }^{\mathbf{b}}$ Department of Vascular Surgery, Qena University Hospital, South Valley University, Qena, Egypt.

\begin{abstract}
Background: Diabetic wounds and ulcers are serious complications in diabetic patients that need a long time and high-cost treatment. There are different physical therapy modalities used in wound care; as Laser therapy, magnetic therapy, negative pressure wound therapy and cryo-air therapy.

Objectives: this study was conducted to investigate the effect of combining therapy between negative pressure wound therapy (NPWT) and cryo-air therapy on diabetic wound healing. Patients and Methods: Sixty diabetic patients with large infected diabetic wounds with age between 40-60 years, positive wound culture after surgical debridement selected and randomly divided into three equal groups, group A received routine medical wound care plus NPWT 3 times/ week for 8 weeks, group B received routine medical wound care plus cryotherapy air 3 times/ week for 8 weeks, group $\mathrm{C}$ received routine medical wound care plus NPWT followed by cryotherapy air application 3 times/ week for 8 weeks. The patients were assessed by wound surface area and wound culture before treatment, after 4 weeks of treatment, and at the end of treatment (after 8 weeks).
\end{abstract}

Results: there was a significant decrease in wound volume at the post I and post II compared with that at pretreatment in the three groups. Also, there was a significant decrease in wound volume at post-II compared with that at the post I in the three groups. Also, there was a significant decrease in the positive culture at the post I compared with that pretreatment in group $\mathrm{B}$ and $\mathrm{C}$ and a significant decrease in the positive culture at the post II compared with pretreatment in group A, B and C. There was a significant decrease in positive wound culture of the group C at post I and post II compared with that of the group A and group B. There was a significant decrease in positive wound culture of the group B compared with that of the group A in the post I and at post II.

Conclusion: cryo-air therapy and negative pressure wound therapy have a positive effect on wound healing but its combination is more effective.

Keywords: Diabetic wounds, negative pressure wound therapy, cryo-air therapy.

Copyright: (C) Elshazly \& Askary et al. (2022) Immediate open access to its content on the principle that making research freely available to the public supports a greater global exchange of knowledge. Users have the right to Read, download, copy, distribute, print or share link to the full texts under a Creative Commons BY-NC-SA 4.0 International License. 


\section{Introduction}

Chronic diabetic wounds and ulcers are one of the big health problems that need a long time and high-cost treatment. Physical therapy contributes to solving this problem by using different devices as LASER, magnetic therapy, hyperbaric oxygen therapy, vacuumassisted closure, and recently cryotherapy air (Turan et al., 2015).

Negative pressure wound therapy (NPWT), also called Vacuum-assisted closure (VAC), has transformed wound care over the last 15 years (Peinemann $\&$ Labeit, 2019).

Dressings to treat chronic wounds have traditionally been gone through several stages as cotton gauze which could be soaked with a variety of chemical substances including normal saline and sodium hypochlorite solutions. In the 1960s, the importance of keeping the wound moist was discovered and a wide array of hydrogels, alginates, and other polymeric and biologically based dressings were developed. More recently, dressing materials have been combined with antimicrobial materials such as silver. Collectively, there are approximately 1500 dressing types available to be used by clinicians in the United States. (Huang et al., 2014)

NPWT improves wound healing by direct effect through micro deformation effect, fluid and exudate removal, and alteration of the wound environment, and by direct effect through modulation of inflammation, cellular responses -division, migration, differentiation, angiogenesis, peripheral nerve response, and alterations in bio-burden (Orgillet al., 2009;Borgquist O, et al., 2011; Scherer et al., 2008).
Cryotherapy air is one of the most recent novel physical therapy devices used in the treatment of infected wounds. It is cheap, easy, accessible, and has fewer side effects in the treatment of chronic wounds. Cryotherapy improves wound healing process through decrease the time of the inflammatory phase, and its bactericidal effect (Somuet.al., 2017).

This study aims to test the combination therapy between negative pressure wound therapy and cryotherapy air on the healing of complex chronic wounds.

\section{Patients and methods \\ Patients}

Sixty diabetic patients with large infected diabetic wounds with age between 40-60 years, positive wound culture after surgical debridement selected from vascular surgery department, south valley university hospital and physical therapy outpatient clinics, faculty of physical therapy, south valley university at the period between July 2019 and January 2020.

\section{The inclusion criteria included:}

- Age: 40- 60 years

- Positive wound culture

- All patients participated in study after surgical debridement.

- Controlled diabetes.

\section{The exclusion criteria included}

- Untreated osteomyelitis within the wound

- non-enteric and unexplored fistula,

- Unconscious patients.

\section{Ethical approval}

This study protocol was approved by ethics of committee for experimental 
involving humans at South Valley University.

\section{Randomization}

After the patients received a clear explanation of the treatment application, they signed a written informed consent. To

\section{Procedures}

\section{Assessment procedures:}

\section{1- Wound volume:}

- Wash and sterilize your hands.

- Apply gloves.

- Remove wound dressing.

- Clean and remove wound debris by saline.

- Sterilize the tape or ruler will be used in measurements.

- Measure the wound longest area in $12^{\circ}$ clocks to $6^{\circ}$ clock directions.

- Measure the breadth in $3^{\circ}$ clocks and $9^{\circ}$ clock at the widest part perpendicular to the length.

- Lastly, apply these measurements in this formula:

Area $=$ length $(\mathrm{L}) \mathrm{X}$ width $(\mathrm{W}) \mathrm{X}$ depth (D) of the wound. (Khoo\& Jansen., 2016)

\section{2- Wound culture:}

Swab culture: It is a laboratory test applied to identify the type of bacteria that causing the wound infection and guiding the line of treatment. It is recommended when there are signs and symptoms of infection (tenderness, swelling, hotness, redness).

Procedure: randomly allocate patient into group A,B and $\mathrm{C}$ block randomization method applied. Patients were blinded about which group they are allocated to, study or control.

- Wash and sterilize your hands, apply gloves and remove the wound dressing.

- Clean the wound and remove excess debris by using normal saline.

- Apply new gloves.

- Moisten the swab with $0.9 \%$ sodium chloride.

- Insert swab into the small clean area of the wound and rotate it for 5 seconds (avoid necrotic areas and edges of the wound).

- Insert swab into a sterilized container.

- Apply the wound dressing and sterilize your hands.(Haalboom, et al., 2019)

\section{Treatment procedures:}

Group A received routine medical wound care plus NPWT 3 times/ week for 8 weeks, group B received routine medical wound care plus cryotherapy air 3 times/ week for 8 weeks, group $\mathrm{C}$ received routine medical wound care plus NPWT followed by cryotherapy air application 3 times/ week for 8 weeks. The patients were assessed by wound surface area and wound culture before treatment, after 4 (post I) weeks of treatment, and at the end of treatment (after 8 weeks) (post II).

\section{a- Surgical debridement}

All operations were done in the operation room. Patients were subjected to local anesthesia to be done by the same vascular surgeon. Draining any abscess and 
removal of all infected necrotic nonviable tissues was the aim of surgical debridement, which was done using a surgical blade taking all layers till reaching healthy bleeding tissues. After hemostasis with diathermy, wounds were irrigated with hydrogen peroxide and then normal saline to be dressed after that using sterile gauze.

\section{b-Negative pressure wound therapy} (NPWT):Negative pressure device: VAC system -125 mmHg (KCI negative pressure equipment) that consists of nonadherent sponge dressing, a draining tube placed in the dressing, a transparent film to seal the wound, drainage tube is connected into a source of negative pressure.

\section{The treatment procedures are applied} through the following steps:

- Firstly, the parameters of the NPWT device should be suited with intermittent negative pressure of $-125 \mathrm{mmHg}$, with duration of five minutes on and two minutes off for $24 \mathrm{~h}$.

- Open-pore sterilized foam dressing was gently cut to fit the wound surface. Such sizes of pores provide an even equal distribution of VAC over the entire wound bed to aid in wound healing.

- Then insert a sterilized Evacuation catheter tube into the dressing.

- The dhesive drape is then applied over the wound surface area plus 3-5 $\mathrm{cm}$ border of intact skin to provide an intact seal and securing vacuum.

- Then connect the catheter into the negative pressure device and begin the treatment. (Kundu et al., 2018)
Broad-spectrum antibiotics started immediately after the operation till the result of the culture and sensitivity test, after which the antibiotic was changed according to the organism sensitivity that was identified.

Wound dressing was done within 48 hours after the operation, later on, weekly until wound healing.

\section{c- Cryotherapy procedures:}

cryotherapy device: cryo-air machine, brand mini, made in Germany, cryo-air cold therapy technology is a mature and safe-touse system that cools down air to $32{ }^{\circ} \mathrm{C}$.

Cryotherapy was applied through the following steps

- Reset the machine on level 8.

- The handheld part was applied to the wound surface in a perpendicular direction.

- When the wound color becomes nearly white stop the application and waits till the color return red again and repeat this application 3 times.

- Time of treatment 10 minutes.

\section{d- Combined therapy between NPWT} and cryotherapy:

After application of NPWT for 24 $h$ and removal of the dressing:

- Clean the wound surface.

- Then therapist applied the cryotherapy air on the wound surface. 
- Apply traditional dressing and not remove for $24 \mathrm{~h}$.

\section{Statistical analysis}

Descriptive statistics and ANOVAtest were conducted for comparison of age between groups. Chi-squared test was used for the comparison of sex distribution between groups. The normal distribution of data was checked using the ShapiroWilk test. Levene's test for homogeneity of variances was conducted to ensure homogeneity between groups.MANOVA test was conducted to compare the mean values of wound volume between groups.

\section{Results}

\section{Subject characteristics}

Table (1) showed the mean $\pm \mathrm{SD}$ age of the three groups. There was no significant difference in age between
Repeated measure ANOVA was conducted for comparison between pre, post I, and post II in each group. Chisquared test was used for comparison of wound culture between groups. Cochran and McNemar's tests were conducted for comparison of wound culture across time intervals in each group. The level of significance for all statistical tests was set at $\mathrm{p}<0.05$. All statistical analysis was conducted through the statistical package for social studies (SPSS) version 22 for windows (IBM SPSS, Chicago, IL, USA).

groups $(\mathrm{p}=0.7)$. Also, there was no significant difference in sex distribution between groups $(\mathrm{p}=0.8)$.

Table 1. Comparison of subject characteristics between groups A, B, and C

\begin{tabular}{|lllll|}
\hline & \multicolumn{4}{c}{ mean \pm SD } \\
\cline { 2 - 5 } Age (years) & Group A & Group B & Group C & p-value \\
\cline { 2 - 5 } Males/females & $52.3 \pm 7.94$ & $53.65 \pm 6.06$ & $51.9 \pm 6.61$ & 0.7 \\
& $12 / 8$ & $14 / 6$ & $13 / 7$ & 0.8 \\
\hline
\end{tabular}

SD, Standard deviation; p- value, Probability value.

\section{Effect of treatment on wound volume}

\section{- Within group comparison:}

There was a significant decrease in wound volume at the post I and post II compared with that at pretreatment in the three groups $(\mathrm{p}<0.001)$. Also, there was a significant decrease in wound volume at post-II compared with that at the post I in the three groups $(\mathrm{p}<0.001)$. (Table 2), (Figure 3)

\section{- Between groups comparison:}

There was no significant difference in the wound volume between the three groups pre-treatment $(\mathrm{p}>0.05)$. In the post I, there was a significant decrease in wound volume of group $\mathrm{C}$ compared with that of group A $(p=0.001)$ and group B (p $=0.02)$; and a significant decrease in wound volume of group B compared with that of group A $(p=0.03)$. At post II, there was a significant decrease in wound volume of group $\mathrm{C}$ compared with that of group A $(p=0.001)$ and group $B(p=$ 0.001 ); and a significant decrease in wound volume of group B compared with that of group A $(p=0.002)$. (Table 2), (Figures 1,2,3). 
Table 2. Mean wound volume at pretreatment, the post I and post II of groups A, B, and C

\begin{tabular}{|c|c|c|c|c|c|c|}
\hline \multirow{2}{*}{$\begin{array}{l}\text { Wound volume } \\
(\mathrm{ml})\end{array}$} & Group A & Group B & Group C & p-valu & & \multirow[b]{2}{*}{ B vs C } \\
\hline & mean \pm SD & mean \pm SD & mean $\pm \mathrm{SD}$ & A vs B & A vs C & \\
\hline Pre treatment & $97.13 \pm 14.04$ & $99.87 \pm 11.76$ & $94.11 \pm 13.63$ & 1 & 1 & 0.51 \\
\hline Post I & $79.93 \pm \mathbf{1 3 . 9 8}$ & $68.55 \pm 12.85$ & $56.45 \pm 14.45$ & 0.03 & 0.001 & 0.02 \\
\hline \multirow[t]{2}{*}{ Post II } & $62.12 \pm 11.11$ & $49.67 \pm 9.8$ & $29.75 \pm 11.55$ & 0.002 & 0.001 & $\begin{array}{l}0.00 \\
1\end{array}$ \\
\hline & $\mathbf{p}=\mathbf{0 . 0 0 1}$ & $p=0.001$ & $\mathbf{p}=\mathbf{0 . 0 0 1}$ & & & \\
\hline
\end{tabular}

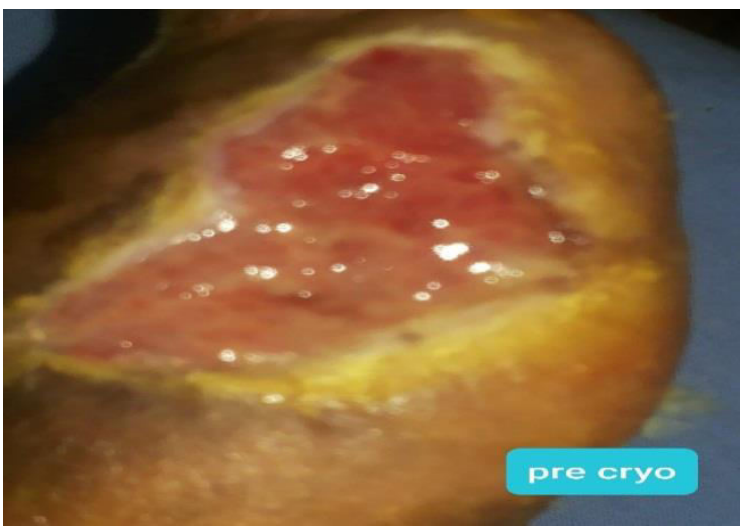

A

This plate shows the volume and appearance of diabetic foot ulcer after big toe amputation before application $\mathrm{f}$ cryotherapy, (volume $=$ $24.75 \mathrm{~cm})$

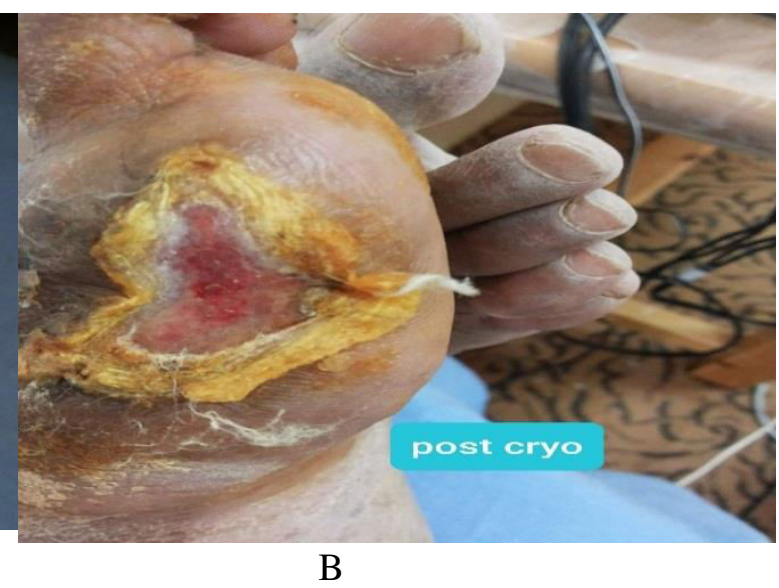

This plate shows the improvement of the wound volume and appearance after cryotherapy treatment. $($ volume $=18.5)$

\section{Fig.1. The differences in wound volume pre and post cryotherapy application}

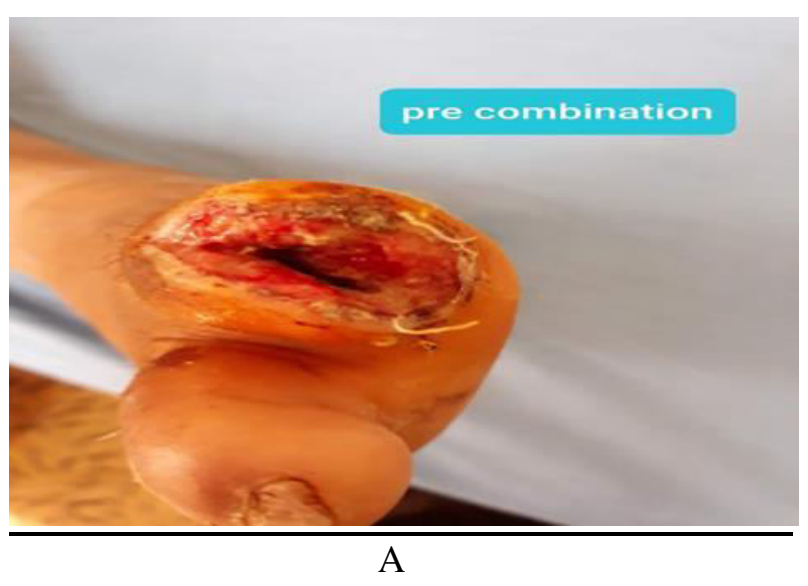

This plate shows wound volume an appearance before application of combination therapy between cryotherapy and NPWT. (volume = $18 \mathrm{~cm})$

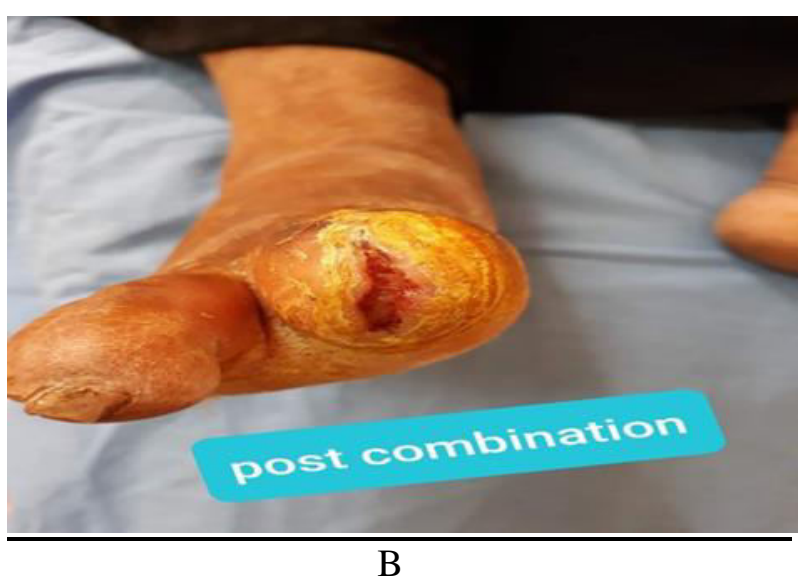

This plate shows wound volume an appearance after application of combination therapy between cryotherapy and NPWT. $($ volume $=4 \mathrm{~cm})$

Fig.2. Wound volume pre and post combination therapy 


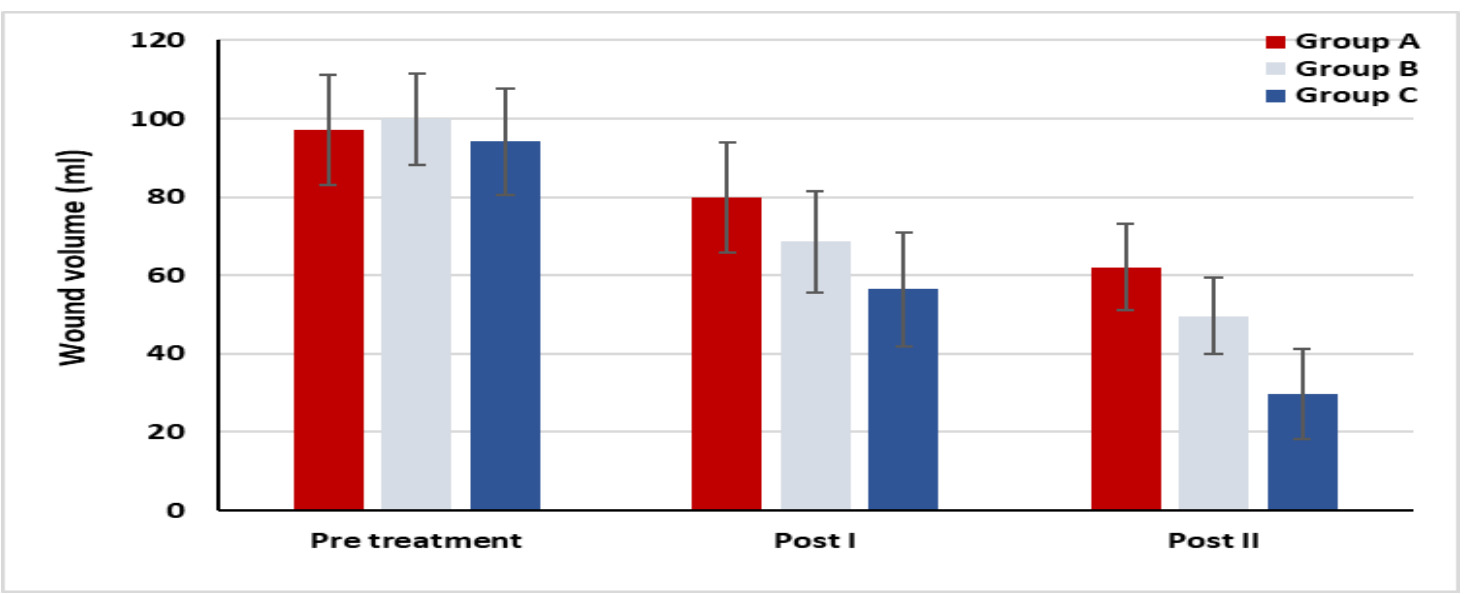

Fig.3. Mean wound volume at pretreatment, the post I, and post II of group A, B and C Effect of treatment on wound culture

- Within-group comparison:

There was a significant decrease in the positive culture at the post I compared with that pretreatment in group B and $\mathrm{C}(\mathrm{p}$ $<0.01$ ) and a significant decrease in the positive culture at post-II compared with pretreatment in groups $\mathrm{A}, \mathrm{B}$, and $\mathrm{C}(\mathrm{p}<$ $0.01)$. There was a significant decrease in the positive culture at post-II compared with that at the post $\mathrm{I}$ in groups A and B (p $<0.01$ ) while there was no significant difference between post I and post II in group C ( $>$ > 0.05), (Table 3).

\section{- Between groups comparison:}

There was a significant decrease in positive wound culture of group $\mathrm{C}$ at the post I and post II compared with that of group A ( $<<0.001)$ and group $B(p<$ 0.01 ). There was a significant decrease in positive wound culture of group B compared with that of group A at the post I and at post II ( $\mathrm{p}<0.05)$ (Table 3$)$.

Table 3. Wound culture at pretreatment, the post I and post II of group A, B, and C

\begin{tabular}{|c|c|c|c|c|c|}
\hline \multirow{3}{*}{$\begin{array}{l}\text { Wound culture } \\
\text { Pre treatment }\end{array}$} & Group A & Group B & Group C & \multirow[b]{2}{*}{$\chi^{2}$ - value } & \multirow[b]{2}{*}{$p$ value } \\
\hline & Frequency & Frequency & Frequency & & \\
\hline & \multirow{3}{*}{$\begin{array}{l}20(100 \%) \\
0(0 \%)\end{array}$} & \multirow[b]{2}{*}{$20(100 \%)$} & \multirow[b]{2}{*}{$20(100 \%)$} & & \\
\hline Positive & & & & \multirow{2}{*}{ - } & \multirow{2}{*}{-} \\
\hline Negative & & $0(0 \%)$ & $0(0 \%)$ & & \\
\hline \multicolumn{6}{|l|}{ Post I } \\
\hline Positive & $20(100 \%)$ & $12(60 \%)$ & $3(15 \%)$ & \multirow{2}{*}{29.86} & \multirow{2}{*}{0.001} \\
\hline Negative & $0(0 \%)$ & $8(40 \%)$ & $17(85 \%)$ & & \\
\hline \multicolumn{6}{|l|}{ Post II } \\
\hline Positive & $12(60 \%)$ & $5(25 \%)$ & $0(0 \%)$ & \multirow{2}{*}{23.1} & \multirow{2}{*}{0.001} \\
\hline Negative & $8(40 \%)$ & $15(75 \%)$ & $20(100 \%)$ & & \\
\hline \multirow{2}{*}{$\begin{array}{l}\chi^{2} \text { - value } \\
\text { p value }\end{array}$} & 16 & 27.44 & 34.9 & & \\
\hline & 0.001 & 0.001 & 0.001 & & \\
\hline
\end{tabular}

$\chi^{2}$, Chi squared value; $p$ value, Probability value. 


\section{Discussion:}

In this study, the effect of VAC therapy, cryotherapy, and combined therapy between VAC and cryotherapy on chronic wounds were tested. After analysis of study data, there was a significant decrease in wound volume at the post I and post II compared with that at pretreatment in the three groups. Also, there was a significant decrease in wound volume at post-II compared with that at the post $\mathrm{I}$ in the three groups. At post I, there was a significant decrease in wound volume of group $\mathrm{C}$ compared with that of group $\mathrm{A}$ and group $\mathrm{B}$; and a significant decrease in wound volume of group B compared with that of group A. in the post II, there was a significant decrease in wound volume of group $\mathrm{C}$ compared with that of group A and group B; and a significant decrease in wound volume of group B compared with that of group A.

Also, there was a significant decrease in the positive culture at the post I compared with that pretreatment in group $\mathrm{B}$ and $\mathrm{C}$ and a significant decrease in the positive culture at post-II compared with pretreatment in group $\mathrm{A}, \mathrm{B}$, and $\mathrm{C}$. There was a significant decrease in positive culture at post II compared with that at post $\mathrm{I}$ in group $\mathrm{A}$ and $\mathrm{B}$ while there was no significant difference between post I and post II in group C. There was a significant decrease in positive wound culture of the group $\mathrm{C}$ at post I and post II compared with that of the group A and group B. There was a significant decrease in the positive wound culture of the group B compared with that of the group $\mathrm{A}$ at the post I and at post II.

VAC therapy is valuable in the treatment of open musculoskeletal injuries where it decreases wound size, stimulates granulation tissue, and clears the wound from exudate and bacteria. Also use of this therapy appears to decrease the need of flap grafting for soft tissue defects. (Kundu et al., 2018)

NPWT causes a reduction in bacteria due to multiple factors: 1removing excess wound exudates and fluid and its positive effect on local blood and lymphatic flows, 2-greater amounts of oxygen made available for the bacterial killing oxidative bursts and the closed nature of the system. The authors also showed a drastic decrease in infection by using VAC therapy when compared with saline dressing leading to a decrease in hospital stay time and decrease numbers of debridement required and reduce morbidity rate (Mehbod et al., 2005).

A systematic review revealed that NPWT decrease the healing time of lower limb ulcers but there is no evidence on the effectiveness of NPWT as primary leg ulcers wound healing treatment (Dumville, et al. 2015)

The growth of bacterial organisms is depressed by lowering the temperature, but it is important to remember that in most instances bacteria can be kept alive for long periods at temperatures of 50 to I00 C. without being damaged or destroyed," whereas, prolonged cooling of an extremity of any of the higher animals or man leads to certain harmful effects on the tissues (Assadian et al., 2019).

Wound cooling would maintain and improve microcirculation that leading to better wound healing and contraction (Yanagisawa, et al., 2007).

Cryotherapy in the form of ICE massage around the surface of the wound 
promoted pain relief and wound contraction faster in the experimental group than in the control group reduces pain and speeds up the wound healing when compared to the normal. Hencethereby we reject the null hypothesis (Potturi and Reddy, 2014).

\section{Conclusion}

From the gained results it could be concluded that NPWT and cryoair therapy are effective in treatment of the post burn scar, but the combination treatment between NPWT and cryoair therapy is more effective. The effectiveness of combined therapy coming from ability of NPWT in the filtration of the wounds from exudate and debris, and the ability of the cryoair therapy in destroying the bacteria and decrease its growth.

\section{Conflict of interest:}

Authors have no conflict of interest to report.

\section{References}

1- Turan Y, Ertugrul B M, Lipsky B A, Bayraktar K. (2015). Does physical therapy and rehabilitation improve outcomes for diabetic foot ulcers?. World journal of experimental medicine, 5(2), 130-139.

2- Peinemann F, Labeit A. (2019): Negative pressure wound therapy: A systematic review of randomized controlled trials from 2000 to 2017; J Evid Based Med, 12:125-132.

3- Huang C, Leavitt T, Bayer LR. (2014): Effect of negative pressure wound therapy on wound healing. current problems in surgery, 51:301331.

4- Orgill D, Manders E, Sumpio B, Lee R, (2009): Themechanisms of action of vacuum-assisted closure: more to learn. Surgery, 146 (1):40-51.
5- Borgquist O, Ingrmansson R, Malmsij M. (2011): The influence of low and high-pressure levels during negative-pressure wound therapy on wound contraction and fluid evacuation. PlastReconstr Surg, 127(2):551-559

6- Scherer SS, Pietramaggiori G, Mathews JC, Prsa MJ, Huang S , Orgill D. (2008). The mechanism of action of the vacuum-assisted closuredevice. PlastReconstrSurg, 122(3):786-797.

7- Agalya V S, Sathivel A, Vaijayanthimala M., (2017). effect of cryotheray on pain perception and wound healing among mothers with episiotomy, International Journal of Science and Research (IJSR),6 (12); 399-409.

8- Rachel K, Shirley J. (2016). The Evolving Field of wound measurement techniques: A Literature Review, WOUNDS journal, 28(6): 175-181.

9- Haalboom M, Blokhuis-Arkes MHE, Beuk RJ, Meerwaldt R, Klont R, Schijffelen MJ, et al. (2019). Culture results from wound biopsy versus wound swab: does it matter for the assessment of wound infection?. Clinical Microbiology and infection, 25(5):629.e7-629.e12.

10-Kundu A, Phuljhele S, Wale N, Gurudatta HS, Agrawal V. (2018). Study of effects of negative pressure wound therapy in contaminated wounds. Int J Res Orthop, 4(5):803807.

11- Mehbod AA, Ogilvie JW, Pinto MR, Schwender JD, Transfeldt EE, Wood KB, et al.. (2005). Postoperative deep wound infections in adults after spinal fusion:Management 
with Vacuum-Assisted Wound Closure. J Spinal Disord Tech, 18:147.

12-Dumvill JC, Land L, Evans D, Peinemann F. (2015). Negative pressure wound therapy for treating leg ulcer.CochranceDatabase Syst Rev, (7): CD011354.

13-Assadian O, Ousey KJ, Daeschlein G, Kramer A, Parker C, Tanner J, et al. (2019). Effect and safety of atmospheric low-temperature plasma on bacterial reduction in chronic wounds and wound size reduction: A systematic review and meta-analysis. Int Wound J, 16(1): 103-111.

14- Yanagisawa O, Homma T, Okuwaki T, Shimao D, Takahashi H. (2007).Effect of cooling on human skin and skeletal muscle. Eur $\mathbf{J}$ ApplPhsiol, 100(6):737-45.

15-Potturi G, Reddy VK. (2014). Effect of cryotherapy on pain and wound healing in the form of standardised ice massage around the surface of wounds in diabetic patients: a randomized control trail, IJBPAS, 3(2): 174-182. 